\title{
Light nuclei in galactic globular clusters: constraints on the self-enrichment scenario from nucleosynthesis
}

\author{
N. Prantzos ${ }^{1}$, C. Charbonnel ${ }^{2,3}$, and C. Iliadis ${ }^{4}$ \\ 1 Institut d'Astrophysique de Paris, UMR7095 CNRS, Univ. P. \& M. Curie, 98bis Bd. Arago, 75104 Paris, France \\ e-mail: prantzos@iap.fr \\ 2 Geneva Observatory, University of Geneva, chemin des Maillettes 51, 1290 Sauverny, Switzerland \\ e-mail: Corinne.Charbonnel@obs. unige.ch \\ 3 Laboratoire d'Astrophysique de Toulouse et Tarbes, CNRS UMR 5572, OMP, Université Paul Sabatier 3, 14 Av. E. Belin, \\ 31400 Toulouse, France \\ 4 Dpt. of Physics and Astronomy, University of North Carolina, Chapel Hill, NC 2759-3255, USA \\ e-mail: iliadis@unc.edu
}

Received 31 January 2007 / Accepted 26 April 2007

\section{ABSTRACT}

\begin{abstract}
Aims. Hydrogen-burning is the root cause of the star-to-star abundance variations of light nuclei in Galactic globular clusters (GC). In the present work we constrain the physical conditions that gave rise to the observed abundance patterns of $\mathrm{Li}, \mathrm{C}, \mathrm{N}, \mathrm{O}, \mathrm{Na}, \mathrm{Mg}, \mathrm{Al}$, as well as Mg isotopes in the typical case of NGC 6752.

Methods. We perform nucleosynthesis calculations at constant temperature, adopting realistic initial abundances for the proto-cluster gas. We use a detailed nuclear reaction network and state-of-the-art nuclear reaction rates.

Results. Although simplistic, our analysis provides original results and new constraints on the self-enrichment scenario for GCs. Our parametric calculations allow us to determine a narrow range of temperature where the observed extreme abundances of all light elements and isotopes in NGC 6752 are nicely reproduced simultaneously. This agreement is obtained after mixing of the $\mathrm{H}$-processed material with $\sim 30 \%$ of unprocessed gas. We show that the observed C-N, O-Na, Mg-Al, Li-Na and F-Na anticorrelations as well as the behaviour of the $\mathrm{Mg}$ isotopes can be recovered by assuming mixing with various dilution factors. Li production by the stars that build up the other abundance anomalies is not mandatory in the case of NGC 6752.

Conclusions. Observations of $\mathrm{O}, \mathrm{Na}, \mathrm{Mg}$ and $\mathrm{Al}$ constrain the temperature range for $\mathrm{H}$-burning; such temperatures are encountered in the two main candidate "polluters" proposed for GCs, namely massive AGBs and the most massive main-sequence stars. Furthermore, observations require dilution of $\mathrm{H}$-burning processed material with pristine one. They provide no clue, however, as to the nature of the unprocessed material required for mixing. The complementary observations of the fragile Li and F clearly point to ISM origin for the mixed material.
\end{abstract}

Key words. stars: abundances - galaxies: abundances - globular clusters: general - globular clusters: individual: NGC 6752

\section{Introduction}

Clues on the early evolution of Galactic globular clusters (hereafter GCs) may be found in the peculiar abundance patterns of their long-lived low-mass stars that have been intensively studied since the early seventies ${ }^{1}$. Inside any well-studied individual GC these stars present indeed large abundance variations of the light nuclei from $\mathrm{C}$ to $\mathrm{Al}$, although their content in heavier elements (i.e., Fe-group, $\alpha$-elements etc.) appears to be fairly homogeneous $^{2}$.

$\mathrm{H}$-burning through the $\mathrm{CNO}$-cycle and the $\mathrm{NeNa}$ - and $\mathrm{MgAl}-$ chains was identified as the root cause of the chemical inhomogeneities of light nuclei in GCs (Kudryashov \& Tutukov 1988; Denisenkov \& Denisenkova 1989, 1990; Langer et al. 1993;

1 We refer to Gratton et al. (2004) and Sneden (2005) for extensive reviews of the very broad literature related to the chemical properties of GCs; see also references in Prantzos \& Charbonnel (2006, hereafter PC06)

2 This is not the case of $\omega$ Cen which is not a typical GC but may be considered as the surviving remnant of a larger system (e.g. Smith 2004 and references therein).
Langer \& Hoffman 1995). High-resolution spectroscopic analyses performed with very large telescopes revealed that the O-Na and $\mathrm{Mg}-\mathrm{Al}$ anticorrelations discovered first among evolved GC stars on the red giant branch are also exhibited by subgiant and turnoff stars $^{3}$. This extension of the abundance anomalies to unevolved or scarcely evolved GC stars was known for long regarding the $\mathrm{C}-\mathrm{N}$ anticorrelation. It is crucial, because it provides compelling evidence that these patterns cannot be due to in situ nuclear reactions ${ }^{4}$, but that they were already present in the material out of which the observed stars formed. This is the socalled self-enrichment scenario that implies (1) pollution of the intracluster gas by $\mathrm{H}$-processed material ejected by relatively

\footnotetext{
Note that the corresponding large star-to-star abundance variations have never been observed among field stars with comparable metallicity $[\mathrm{Fe} / \mathrm{H}]$, age, and evolutionary status (Hanson et al. 1998; Gratton et al. 2000; Palacios et al. 2002; Mishenina et al. 2006). Environment, therefore, seems to have a crucial influence on the occurrence of these chemical patterns.

${ }^{4}$ In low-mass turnoff stars the temperature is not high enough to allow the proton-captures reactions in the $\mathrm{NeNa}$ - and $\mathrm{MgAl}$-chains as will be discussed later on in more details.
} 
massive and rapidly evolving stars in the early stages of the cluster life (Cottrell \& Da Costa 1981; Smith \& Norris 1982) and (2) formation of a second generation of stars by the ejecta or by a mixture of the ejecta with pristine material. Observations suggest that all metals heavier than Si were already implanted in the proto-cluster gas (i.e., their present abundance is pristine) and that inside the GC itself only the abundances of elements up to Al have been modified with respect to the initial cluster composition (which is the composition of contemporary halo field stars, see PC06 for more details).

The stellar sources responsible for the GC chemical anomalies have not been indubitably identified yet. As noticed by several authors, understanding the most extreme abundances exhibited by stars in GCs is a major key to understand the overall abundance patterns. Several important theoretical efforts have been made towards that goal, with custom-made stellar evolution models of various degrees of sophistication. Competing nucleosynthesis sites today are massive AGB stars on the one hand (Ventura et al. 2001; D'Antona et al. 2002; Denissenkov \& Herwig 2003), and fast rotating massive stars on the other (PC06; Decressin et al. 2007). Both candidate polluters present advantages and problems, not only from the point of view of nucleosynthesis, but also regarding for instance the required numbers of progenitor stars (i.e. the initial mass function of the $1 \mathrm{st}$ generation) or the existence of a triggering process to ignite the formation of the chemically peculiar low-mass stars of the 2nd generation (see PC06 for more details). In both cases the complexity of the involved physical phenomena certainly requires deeper insight.

In view of the important uncertainties still affecting stellar nucleosynthesis models we decided to tackle the problem from a different angle. In the present work we discuss the case of NGC 6752, which is one of the best studied GC regarding its chemical properties; these properties are presented in Sect. 2. In Sect. 3 we aim at finding whether, starting with realistic initial abundances for the protocluster gas, one may reproduce the observed extreme abundances of all the light nuclei for some narrow range of temperature and timescale of H-burning. For that purpose we perform nucleosynthesis calculations at constant temperature, which is a satisfactory approximation for the quiescent stellar burning stages that we have in mind (i.e. central H-burning for massive, mass losing, stars and hot-bottom burning in massive AGB stars). We then explore the dilution factors between $\mathrm{H}$-processed and unprocessed material that are required to reproduce the $\mathrm{C}-\mathrm{N}, \mathrm{O}-\mathrm{Na}, \mathrm{Mg}-\mathrm{Al}$ anticorrelations as well as the behaviour of the $\mathrm{Mg}$ isotopes in NGC 6752. In Sect. 4 we discuss the case of the anticorrelations observed between $\mathrm{Na}$ and the light nuclei Li and F. Based on the strong constraints on temperature that we get from our nucleosynthesis calculations, we discuss in Sect. 5 the corresponding astrophysical sites. In Sect. 6 we summarize our results.

\section{Abundances in NGC 6752}

NGC 6752 is a nearby GC that has been extensively studied in the literature (see Carretta et al. 2007 for references). It is the first GC where the O-Na anticorrelation has been discovered among turnoff stars (Gratton et al. 2001). The published values for its metallicity $[\mathrm{Fe} / \mathrm{H}]$ range between $\sim-1.4$ and -1.6 (e.g., Pritzl et al. 2005). Here we adopt $[\mathrm{Fe} / \mathrm{H}]=-1.5$.

In this work, we consider two sets of abundances for the stars of NGC 6752. The first one concerns the abundances of $\mathrm{C}, \mathrm{N}, \mathrm{O}$ and $\mathrm{Na}$, measured by Carretta et al. $(2005)^{5}$ on high resolution ( $R \sim 40000)$ UVES/VLT spectra; we keep only the measurements of the 8 dwarf stars of the sample, assuming it highly unlikely that they are contaminated by nuclearly processed material from their inner layers (Specifically the surface abundances of $\mathrm{C}$ and $\mathrm{N}$ are modified on the early RGB due to the first dredgeup of internally CN-processed matter; e.g., Charbonnel 1994; Charbonnel et al. 1998; Gratton et al. 2000). The second sample consists of abundances of $\mathrm{O}, \mathrm{Na}, \mathrm{Al}, \mathrm{Mg}$, as well as $\mathrm{Mg}$ isotopes $\left({ }^{24} \mathrm{Mg},{ }^{25} \mathrm{Mg},{ }^{26} \mathrm{Mg}\right)$, measured on very high resolution $(R \sim 110000)$ UVES/VLT spectra by Yong et al. (2003), in 20 bright giant stars (Note that the surface abundances of these heavier elements are not modified during the first dredgeup event nor later on the RGB; e.g., Palacios et al. 2006).

The adopted abundances for NGC 6752 are displayed in Fig. 1, as a function of the $\mathrm{O}$ abundance. $\mathrm{Na}, \mathrm{Al}$ and ${ }^{26} \mathrm{Mg}$ clearly increase with decreasing $\mathrm{O}$ values, $\mathrm{Mg}$ and ${ }^{24} \mathrm{Mg}$ decrease (slightly), while ${ }^{25} \mathrm{Mg}$ appears to be insensitive to $\mathrm{O}$ variations (as already discussed in Yong et al. 2003). We note that the variation of oxygen abundance is larger in the case of Carretta et al. (2005, a factor of $\sim 8)$ than in the case of Yong et al. (2003, a factor of 5). In the former case, indeed, the authors have tried and enhanced the chance of finding extreme cases by selecting stars with likely strong and weak $\mathrm{CN}$ bands using the Strömgren $c_{1}$ index.

The largest $\mathrm{O}$ values are similar to the ones of field halo stars of the same metallicity as NGC 6752; we assume that this is the original $\mathrm{O}$ abundance of the intracluster gas. This is also true for the corresponding values of $\mathrm{Na}, \mathrm{Al}$ and $\mathrm{Mg}$. We consider then that all abundances corresponding to the highest $\mathrm{O}$ abundances (as indicated by open circles in Fig. 1) are the initial ones and that abundances at the other extreme (indicated by open squares) correspond to the most extremely processed material. For the initial abundances of $\mathrm{C}$ and $\mathrm{N}$, we adopt $[\mathrm{C} / \mathrm{N}]$ and $[\mathrm{N} / \mathrm{Fe}]=0$, in view of the corresponding abundances of field halo stars (Spite et al. 2005). We note that our adopted initial abundances for $\mathrm{Mg}$ isotopes, based on observations of NGC 6752 (Fig. 1, right panels) do not correspond exactly to the scarce available observations of field halo stars of the same metallicity. This "mismatch" was noted by Yong et al. $(2003,2006)$ and it was attributed by these authors to the action of a specific class of "Mg-only polluters" (assumed to be AGB stars), beyond the polluters required to explain the other composition anomalies. We do not consider here that idea, which introduces another level of complexity to a subject which is quite complex already. We simply note that (i) only in few field halo stars the $\mathrm{Mg}$ isotopic ratios have been measured, and further observations are required before concluding on this (very important) topic; (ii) our adopted initial isotopic ratios are $\operatorname{Mg} 24: 25: 26=87: 6.5: 6.5$ (see Fig. 1 and Table 1) i.e. they differ very little from the ones measured in NGC 6752 by Yong et al. (2003), who give 83:10:7 for the highest ratio (corresponding to normal/unprocessed stars). In any case, those low values do not affect the calculated final abundances of those isotopes or of ${ }^{27} \mathrm{Al}$, which are produced mostly from initial ${ }^{24} \mathrm{Mg}$ in those temperatures.

For clarity, we display in Table 1 the assumed original and most extreme values for $\mathrm{O}, \mathrm{Na}, \mathrm{Al}, \mathrm{Mg},{ }^{24} \mathrm{Mg},{ }^{25} \mathrm{Mg}$ and ${ }^{26} \mathrm{Mg}$,

\footnotetext{
5 Carretta et al. (2007) present the largest sample of $\mathrm{O}$ and $\mathrm{Na}$ abundance determinations to date in NGC 6752. For seek of homogeneity we chose however to use the smaller sample of 2005 for which the abundances of $\mathrm{C}$ and $\mathrm{N}$ were simultaneously determined. This choice does not affect the present discussion.
} 

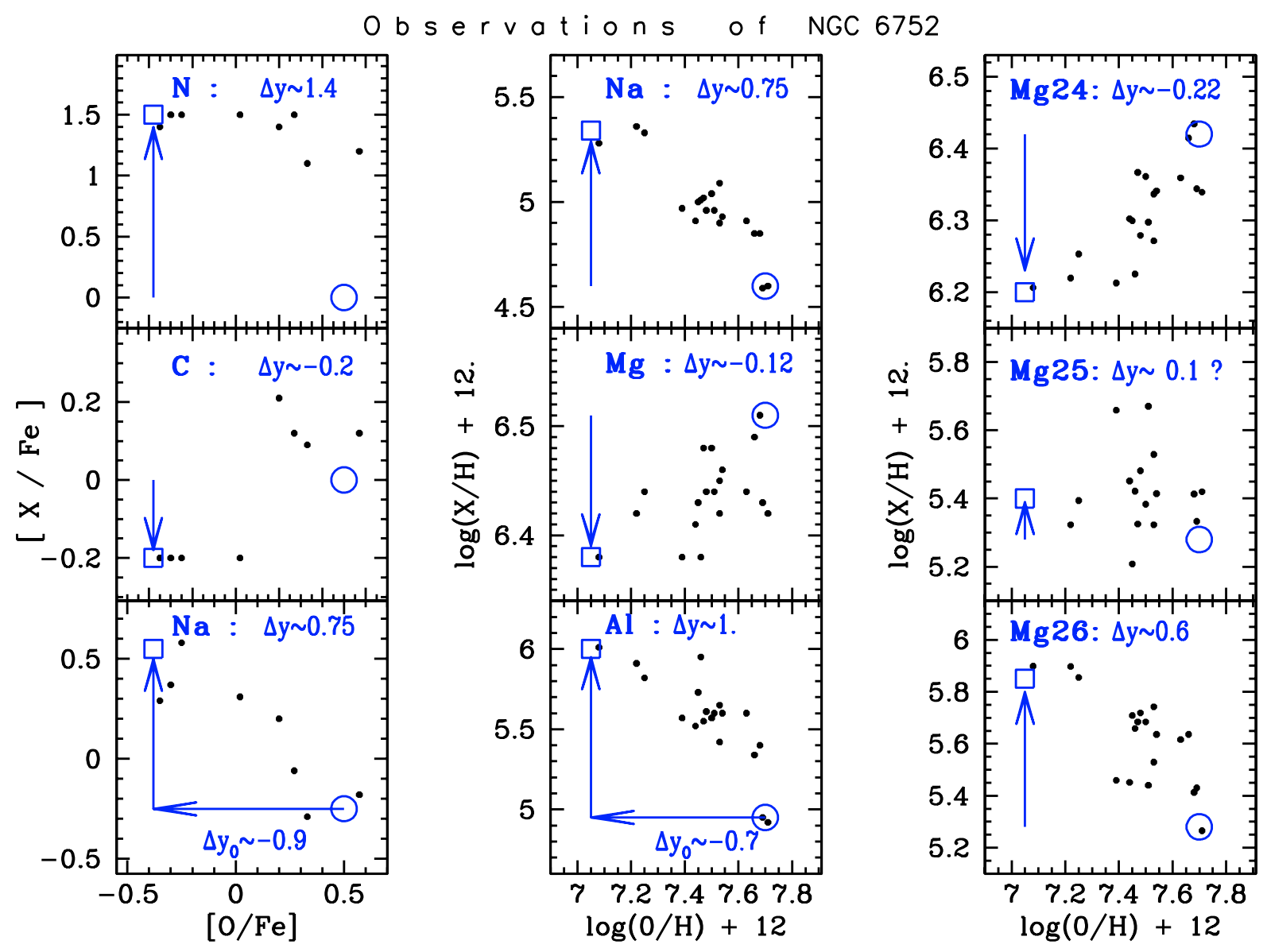

Fig. 1. Abundances of C, N, Na, Mg, $\mathrm{Al}$ and Mg isotopes in stars of NGC 6752, as a function of the corresponding oxygen abundance. Observations (filled symbols) in the left column (plotted as a function of [O/Fe]) are from Carretta et al. (2005), and in the other two columns (plotted as a function of $\mathrm{O} / \mathrm{H}$ ) from Yong et al. (2003). In all the figures, the estimated initial abundances of the gas from which the cluster was formed are indicated by an open circle (i.e., it is assumed that they have the same composition as field halo stars of the same metallicity $[\mathrm{Fe} / \mathrm{H}]=-1.5$, with e.g. $[\mathrm{O} / \mathrm{Fe}]=0.5,[\mathrm{C} / \mathrm{Fe}]=0$, etc.). The most extreme abundances observed at present in NGC 6752 are indicated by open squares. Arrows indicate the magnitude and direction of the abundance spread, which is also given by $\Delta y$ (in dex, where $y=\log \left(\right.$ abundance)) in each panel; $\Delta y_{\mathrm{O}}$ in the bottom panels indicates the corresponding variation of oxygen abundance, larger in the case of Carretta et al. (2005, a factor of $\sim 8)$, than in the case of Yong et al. (2003, a factor of 5).

both by mass fraction and by number (in the $\log \left(N_{\mathrm{H}}\right)=12$. scale), as well as the corresponding $\mathrm{Mg} 24: 25: 26$ ratios.

Our aim is then to find whether, starting with the original abundances $X_{\mathrm{O}}$ (open cicles in Fig. 1), one may reproduce the observed extreme abundances $X_{\mathrm{E}}$ (open squares in Fig. 1) for some, hopefully narrow, range of temperature $T$ and timescale $\tau$ of H-burning.

\section{Nucleosynthesis}

\subsection{The set-up}

\subsubsection{Nuclear reaction network}

We perform H-burning nucleosynthesis calculations at constant temperature $T$. Since all the reactions involved are 2-body reactions, density plays no role in the outcome, it only affects directly the various timescales. For that reason, we display our results as a function of the consumed $\mathrm{H}$ mass fraction (the initial one being $X_{\mathrm{H}}=0.75$ ) and not of time. We perform calculations for temperatures in the range $T=25$ to $80 \mathrm{MK}$ (millions of degrees) that encompass typical burning conditions in a large variety of realistic stellar models (see Sect. 5).
The adopted reaction network extends from $\mathrm{H}$ to the $\mathrm{Ca}$ region and contains more than 140 nuclei, including very protonrich ones. For instance, the range of $\mathrm{O}$ isotopes extends from ${ }^{13} \mathrm{O}$ to ${ }^{18} \mathrm{O}$ and for the $\mathrm{Mg}$ isotopes from ${ }^{20} \mathrm{Mg}$ to ${ }^{26} \mathrm{Mg}$. It contains all the reactions of the cold and hot pp chains, the cold and hot $\mathrm{CNO}$ cycles, and all relevant interactions involving nuclei in the $\mathrm{NeNa}$ and $\mathrm{MgAl}$ mass ranges. The nuclide ${ }^{26} \mathrm{Al}$ is treated as two distinct species: the ground state ${ }^{26} \mathrm{Al}^{g}$ and the isomeric state ${ }^{26} \mathrm{Al}^{m}$, since those states are not thermalized at the relatively low temperatures of hydrostatic H-burning studied here. The communication between those states via gamma-ray transitions involving higher-lying ${ }^{26} \mathrm{Al}$ levels is explicitly taken into account in our network. The required gamma-ray transition probabilities are adopted from Runkle, Champagne \& Engel (2001).

Our reaction rate library is based on the evaluations of Angulo et al. (1999) and Iliadis et al. (2001) for the mass ranges of $A<20$ and $A \geq 20$, respectively, with a few exceptions. For the ${ }^{14} \mathrm{~N}(\mathrm{p}, \gamma){ }^{15} \mathrm{O}$ reaction we use the recent rate of Runkle et al. (2005) which is significantly lower than the rate given in Angulo et al. (1999). New experimental information is also available for ${ }^{17} \mathrm{O}(\mathrm{p}, \gamma){ }^{18} \mathrm{~F}$ and ${ }^{17} \mathrm{O}(\mathrm{p}, \alpha){ }^{14} \mathrm{~N}$. Our rates for these reactions are based on Fox et al. (2005) and Chafa et al. (2005), respectively. Finally, the ${ }^{23} \mathrm{Na}(\mathrm{p}, \gamma)^{24} \mathrm{Mg}$ 
Table 1. Observed extreme abundances in NGC 6752.

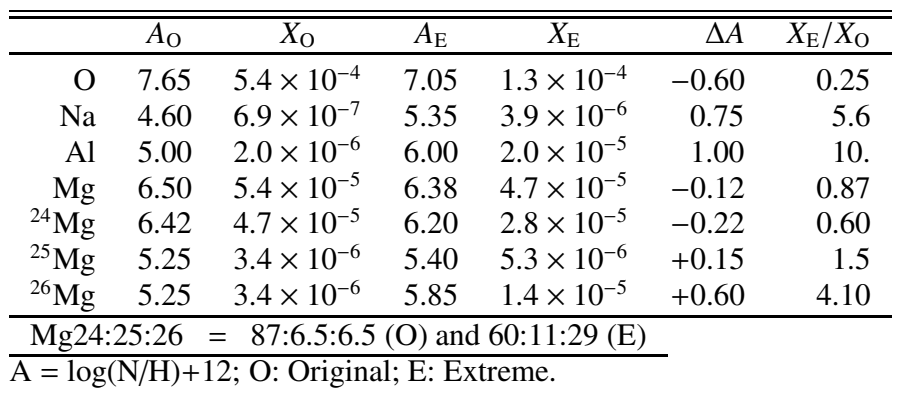

and ${ }^{23} \mathrm{Na}(\mathrm{p}, \alpha){ }^{20} \mathrm{Ne}$ reaction rates are adopted from Rowland et al. (2004). Note that the recommended rates for some of these reactions deviate strongly from the NACRE evaluation (Angulo et al. 1999). For example, at $80 \mathrm{MK}$ the new rates for ${ }^{22} \mathrm{Ne}(\mathrm{p}, \gamma)^{23} \mathrm{Na}$ and ${ }^{23} \mathrm{Na}(\mathrm{p}, \gamma)^{24} \mathrm{Mg}$ are factors of 103 and 33, respectively, smaller than the NACRE rates, while the new ${ }^{27} \mathrm{Al}(\mathrm{p}, \alpha){ }^{24} \mathrm{Mg}$ rate is a factor of 10 higher than reported by NACRE.

\subsubsection{Initial composition}

The initial composition of the mixture is the one of field halo stars of the same metallicity, $[\mathrm{Fe} / \mathrm{H}]=-1.5$, as NGC 6752 (see Sect. 2 and Table 1). The abundances of the light isotopes are those resulting from Big Bang nucleosynthesis, with a ${ }^{7} \mathrm{Li}$ abundance of $\log (\mathrm{Li} / \mathrm{H})+12=2.65$ (after BBM+WMAP; Steigman 2006). The abundances of $\mathrm{ONaMgAl}$ are those suggested by the most O-rich stars in NGC 6752, namely those having $[\mathrm{O} / \mathrm{Fe}] \sim 0.5$ (open circles in Fig. 1), while for $\mathrm{C}$ and $\mathrm{N}$ we adopt $[\mathrm{X} / \mathrm{Fe}]=0$. For the initial isotopic abundances, we adopt the values of Fig. 1 (open circles) for the Mg isotopes (see also Table 1); observational data are not available for other isotopes, and we rely on Galactic chemical evolution calculations (Goswami \& Prantzos 2000) in order to evaluate the corresponding abundances at $[\mathrm{Fe} / \mathrm{H}]=-1.5$.

\subsection{Evolution of the composition during $\mathrm{H}$-burning at constant temperature}

Results of our calculations for six different values of the temperature appear in Fig. 2. The abundances of selected $\mathrm{CNONeNaMgAl}$ isotopes are plotted as a function of consumed mass fraction of hydrogen $\left(X_{0}-X\right) / X_{0}$. In the bottom row of that figure, the abundance ratios (by number) of $\mathrm{C} / \mathrm{O}, \mathrm{Al} / \mathrm{Na}$ and $\mathrm{Na} / \mathrm{O}$ are also displayed.

In the top row it is seen that ${ }^{16} \mathrm{O}$ reaches its equilibrium value at $T=25 \mathrm{MK}$ after a few per cent of initial $\mathrm{H}$ is consumed, and at higher $T$ after $\sim 1 \%$ of $\mathrm{H}$ is consumed; that equilibrium value is slightly temperature dependent. Isotopes involved in the $\mathrm{CN}$ cycle reach, of course, their equilibrium values much earlier.

In the second row, it is seen that ${ }^{23} \mathrm{Na}$ is produced through the destruction of ${ }^{22} \mathrm{Ne}$ already at $25 \mathrm{MK}$. As temperature increases, a fraction of ${ }^{20} \mathrm{Ne}$ (less than $20 \%$ ) is converted to ${ }^{22} \mathrm{Ne}$ and ${ }^{23} \mathrm{Na}$, which reach equilibrium above $T \sim 50 \mathrm{MK}$. The equilibrium value of ${ }^{23} \mathrm{Na}$ is highest around $T=50 \mathrm{MK}$ and decreases slowly at higher $T$; at high temperatures, the equilibrium value of ${ }^{22} \mathrm{Ne}$ remains $\sim$ constant $\sim 0.1$ of ${ }^{20} \mathrm{Ne}$.

${ }^{24} \mathrm{Mg}$ is affected only above $70 \mathrm{MK}$, and only $1 \%$ of its initial abundance is left at $T=80 \mathrm{MK}$. At early times (when less than a few $\%$ of $\mathrm{H}$ is consumed), its destruction leads to an increase of the abundances of ${ }^{25} \mathrm{Mg}$ and ${ }^{26} \mathrm{Al}$. When more than $10 \%$ of $\mathrm{H}$ is consumed, almost all initial ${ }^{24} \mathrm{Mg}$ has turned into ${ }^{27} \mathrm{Al}$, the abundance of which increases by more than a factor of 20 .

The two heavier $\mathrm{Mg}$ isotopes are more fragile than ${ }^{24} \mathrm{Mg}$ and they are destroyed at low temperatures. When ${ }^{24} \mathrm{Mg}$ is affected (above $70 \mathrm{MK}){ }^{25} \mathrm{Mg}$ increases slightly early on, while ${ }^{26} \mathrm{Mg}$ remains $\sim$ constant; at late times, they are both destroyed, following ${ }^{24} \mathrm{Mg}$. Between the two epochs, substantial amounts of ${ }^{26} \mathrm{Al}$ are produced. None of the $\mathrm{Mg}$ and $\mathrm{Al}$ isotopes reaches equilibrium, even when most of $\mathrm{H}$ is consumed.

The production of ${ }^{26} \mathrm{Al}$ may be of importance for ${ }^{26} \mathrm{Mg}$, which is never produced as such (at least with the adopted set of reaction rates). If nuclear reactions cease (because material is brought to lower temperatures, through e.g. convection and stellar winds), then ${ }^{26} \mathrm{Al}$ will decay to ${ }^{26} \mathrm{Mg}$ within $1 \mathrm{Myr}$, and the abundance of the latter will be found substantially enhanced. Note, however, that the ${ }^{26} \mathrm{Mg} /{ }^{24} \mathrm{Mg}$ ratio increases anyway, just because ${ }^{24} \mathrm{Mg}$ is always destroyed.

The corresponding evolution of abundance ratios (by number) of $\mathrm{C} / \mathrm{O}, \mathrm{Na} / \mathrm{O}$ and $\mathrm{Al} / \mathrm{Na}$ is displayed in the bottom row of Fig. 2. It is seen that at high temperatures (above $60 \mathrm{MK}$ ) $\mathrm{C} / \mathrm{O}$ and $\mathrm{Na} / \mathrm{O}$ reach equilibrium values after a few $\%$ of $\mathrm{H}$ is consumed, while enhanced $\mathrm{Al} / \mathrm{Na}$ values are obtained only above $T=70 \mathrm{MK}$.

In summary, our calculations show clearly that in the temperature range $70-80 \mathrm{MK}$ the extreme abundances observed in NGC 6752 can be qualitatively reproduced during the early $\mathrm{H}-$ burning, i.e. after a few $\%$ of $\mathrm{H}$ is consumed. $\mathrm{O}$ is always strongly depleted. ${ }^{24} \mathrm{Mg}$ is also depleted, but to a smaller extent. Na, Al and ${ }^{26} \mathrm{Mg}$ are largely enhanced (the latter in the form of ${ }^{26} \mathrm{Al}$ ). ${ }^{25} \mathrm{Mg}$ is very little affected in that part of H-burning, but decreases considerably as more $\mathrm{H}$ is consumed. Finally, the sum of $\mathrm{Mg}$ isotopes $\left({ }^{24} \mathrm{Mg}+{ }^{25} \mathrm{Mg}+{ }^{26} \mathrm{Mg}+{ }^{26} \mathrm{Al}\right)$ is slightly smaller than the initial $\mathrm{Mg}$ amount, since some leakage takes place out of the $\mathrm{MgAl}$ chain.

We would like to emphasize that in this work we only consider recommended reaction rates. The current rate uncertainties vary strongly from reaction to reaction. For example, at $80 \mathrm{MK}$ the ${ }^{24} \mathrm{Mg}(\mathrm{p}, \gamma){ }^{25} \mathrm{Al}$ reaction has a rate error of only $16 \%$ which makes it the best known rate among all proton-induced reactions on targets in the mass $A \geq 20$ range (Powell et al. 1999). On the other hand, the rate errors for the reactions ${ }^{22} \mathrm{Ne}(\mathrm{p}, \gamma)^{23} \mathrm{Na}$, ${ }^{23} \mathrm{Na}(\mathrm{p}, \gamma){ }^{24} \mathrm{Mg},{ }^{26} \mathrm{Mg}(\mathrm{p}, \gamma){ }^{27} \mathrm{Al}$ and ${ }^{26} \mathrm{Al}(\mathrm{p}, \gamma){ }^{27} \mathrm{Si}$ amount to more than an order of magnitude at $80 \mathrm{MK}$.

Izzard et al. (2007) present a list of such key reactions and explore the effect of the corresponding uncertainties on the outcome of AGB nucleosynthesis. We plan to perform a similar exploration in the framework of our simple one-zone parametrized models in a future work. Here, we simply note that for the key reaction ${ }^{26} \mathrm{Al}(\mathrm{p}, \gamma)^{27} \mathrm{Si}$ (the latter decaying quasi-instantaneously to ${ }^{27} \mathrm{Al}$ ), Izzard et al. (2007) suggest uncertainties by factors $f=0.5$ to 600 over the value of Iliadis et al. (2001) adopted here. By adopting $f=0.5$ we find that higher temperatures than $80 \mathrm{MK}$ are required to obtain the extreme ${ }^{27} \mathrm{Al}$ values, while for $f=50$ temperatures lower than $70 \mathrm{MK}$ are required; in the latter case, however, we never get enough ${ }^{26} \mathrm{Mg}$ (since there is never enough ${ }^{26} \mathrm{Al}$ ). Such temperatures are outside the temperature range that we determine in the next section. In view of that, one may conclude either that:

(i) the true reaction rate values are close to those adopted here, which lead to results agreeing with observations for the temperature range $T \sim 74 \mathrm{MK}$ (see next section), or 


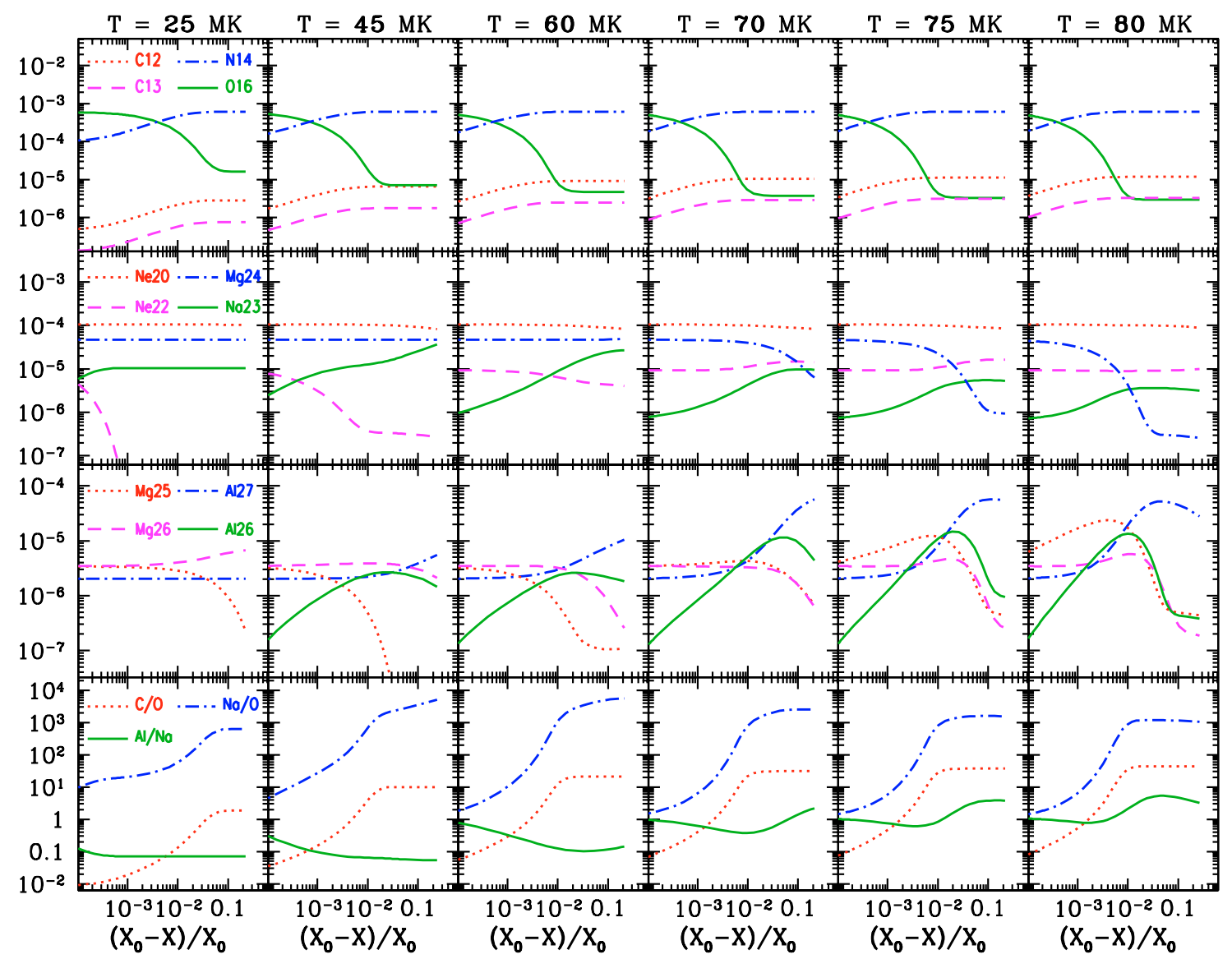

Fig. 2. Evolution of the composition during H-burning at constant temperature. Adopted temperatures are given on top of each column. Results are plotted as a function of the consumed $\mathrm{H}$ fraction $\Delta X / X_{0}$ (where $X_{0}=0.75$ ), i.e. time increases to the right of each panel. Abundances are mass fractions in the first three rows, while in the bottom row the quantity $(A / B) /(A / B)_{0}$ is given (where $A$ and $B$ are abundances by number and 0 stands for abundances in the original mixture).

(ii) some other combination(s) of reaction rates may also satisfy observations, perhaps for some different temperature range.

We keep option (i) here, and we explore the impact of reaction rate uncertainties on our models in future work.

\subsection{Constraining the temperature range}

After the first (and broadly encouraging) estimates based on the results of Fig. 2, we attempt to constrain more quantitatively our parameter space, using the observables of NGC 6752. From the results displayed in Figs. 1 and 2, we note that:

a) Oxygen is the key element, both for observational reasons (it is extensively observed) and for theoretical ones (its abundance varies widely). The regularity of the observed abundance patterns (abundances vary monotonically with $\mathrm{O}$ and with relatively little scatter) suggests that the $\mathrm{O}$ abundance in the processed matter has reached its equilibrium value (otherwise, considerable dispersion in abundance patterns would be expected); consequently, equilibrium values of $O$ should be adopted from Fig. 2.

b) The equilibrium values of $\mathrm{O}$ at high $T$ are $\sim 100$ times lower than the initial ones; but the observed lowest $\mathrm{O}$ abundances in NGC 6752 are only $\sim 5-8$ times lower than the original value (depending on whether the Carretta et al. (2005) or the Yong et al. (2003) data are used). This means that even the extreme observed abundances result necessarily from a mixture of processed material with original one; in other words material coming directly from the H-burning regions is never observed directly, otherwise $\mathrm{O}$ abundances should be much lower than actually observed. Obviously, intermediate abundance values between $X_{\mathrm{O}}$ and $X_{\mathrm{E}}$ should result from various degrees of such mixing.

c) From the previous (mixing) argument, one concludes that no direct comparison is possible between observations (the most extreme values, to the left of all panels in Fig. 1, or $X_{\mathrm{E}}$ in Table 1) and the theoretical results displayed in Fig. 2. However, by combining theory (i.e. the relatively stable - at high $T$ - equilibrium values of $\mathrm{O}$ abundance $X_{\mathrm{EQU}}$ ) and observations (the observed extreme values of $\mathrm{O} X_{\mathrm{OBS}}$ ) one may derive the required mixing factor $f$; indeed, the observed values being a mixture of one part of processed material with $f$ parts of original one, one has

$X_{\mathrm{OBS}}=\frac{X_{\mathrm{PROC}}+f X_{\mathrm{ORIG}}}{1+f}$

from which

$f=\frac{X_{\mathrm{OBS}}-X_{\mathrm{PROC}}}{X_{\mathrm{ORIG}}-X_{\mathrm{OBS}}}$.

In the case of oxygen one has: for the original value $X_{\mathrm{ORIG}}=$ $5.4 \times 10^{-4}$ (Table 1 ), for the extreme observed value of $\mathrm{O}$ $X_{\mathrm{E}, \mathrm{OBS}}=1.3 \times 10^{-4}$ (Table 1 , for the Yong et al. 2003 data) and for the equilibrium value at $T=70-80 \mathrm{MK} X_{\mathrm{PROC}}=3.5 \times 10^{-6}$ (from Fig. 2). From those values one obtains $f_{\mathrm{O}}=0.31$ for oxygen. Applying that mixing (=dilution) factor to all other theoretically obtained abundances through Eq. (1), one may derive then 

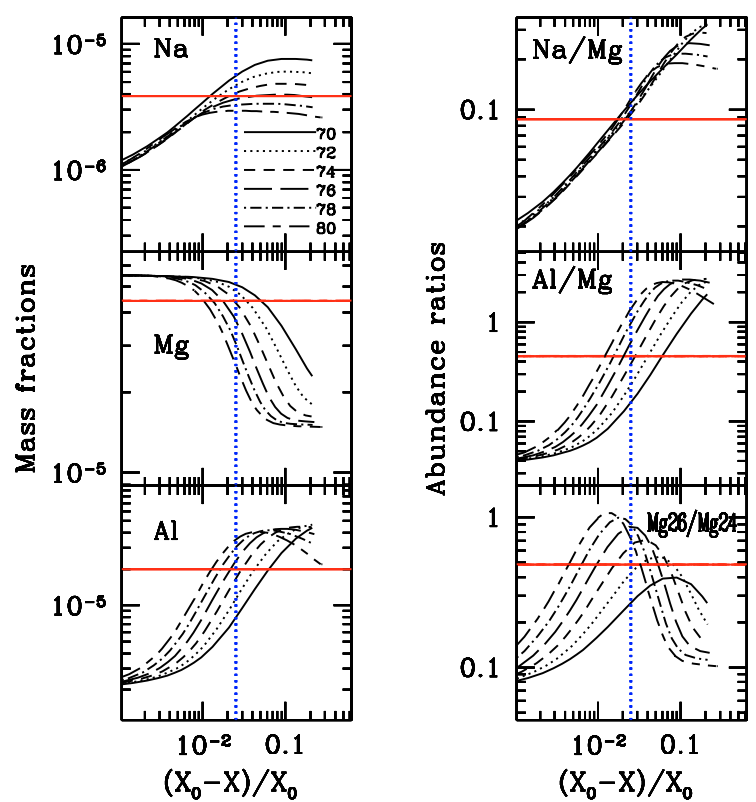

Fig. 3. Evolution of mass fractions (left column) and abundance ratios by number (right column) of elements in the $\mathrm{Na}-\mathrm{Al}$ region, during $\mathrm{H}$ burning at constant temperature; they are plotted as a function of the consumed $\mathrm{H}$ fraction (see Fig. 2) for six values of the temperature in the $70-80 \mathrm{MK}$ range (see top left panel). The abundance of ${ }^{26} \mathrm{Al}$ is always counted in the one of ${ }^{26} \mathrm{Mg}$ and of total $\mathrm{Mg}$. All theoretical values are corrected for mixing with original material with a factor $f=0.31$, such as to ensure that the abundance in the mixture will be equal to $X_{\mathrm{E} \text {,OBS }}$ for oxygen (see text). Horizontal solid lines in all panels represent the observed extreme values of each element or abundance ratio $\left(X_{\mathrm{E}}\right.$ in Table 1). The vertical dotted lines in all panels indicate a consumed $\mathrm{H}$ fraction of $\Delta X / X=0.025$, where calculations at $T=74-76 \mathrm{MK}$ agree with observational requirements for all elements.

the corresponding abundances in the mixed material $X_{\mathrm{E}, \mathrm{OBS}}$ for all other elements of the network. The question then is: are those mixed abundances (which, by construction, reproduce the extreme observed oxygen abundance) compatible with the extreme observed values of all other elements and isotopes appearing in Fig. 1? For what temperatures and consumed fractions of $\mathrm{H}$ ? Is there a range of $T$ and $\Delta X$ satisfying all the observational constraints?

The resulting abundances in the mixture (i.e. the theoretical ones, corrected for mixing with $f=0.31$ ) appear in the various panels of Fig. 3 in a way similar to those displayed in Fig. 2, but this time a more refined grid of temperatures is used, with steps of $\Delta T=2 \mathrm{MK}$ in the temperature range 70 to $80 \mathrm{MK}$. The purpose of that exercice is to determine more precisely the ranges of temperature and consumed $\mathrm{H}$ fraction that satisfy simultaneously all observational requirements; the latter, i.e. the values $X_{\mathrm{E}, \mathrm{OBS}}$ appear as horizontal solid lines in Fig. 3. In other terms, we seek to find whether all the observed extreme abundances in NGC 6752 can be explained in terms of nucleosynthesis occurring in a narrow range of (constant) temperatures, after some mixing with original material. However, since abundances in the $\mathrm{Mg}-\mathrm{Al}$ region do not reach equilibrium but evolve steadily with time (or $\mathrm{H}$ mass fraction), we have to seek for specific solutions in the $T$ vs. $\Delta X$ plane; if all abundances were in equilibrium, the situation would be much simpler and only a range of temperatures would be sought.

An inspection of Fig. 3 shows that $\mathrm{Na}$ is not a good "thermometer", since its quasi-equilibrium abundance varies little (just a factor of 3) between 70 and $80 \mathrm{MK} .{ }^{24} \mathrm{Mg}$ is much more sensitive to temperature: after a few $\%$ of $\mathrm{H}$ is consumed, ${ }^{24} \mathrm{Mg}$ / abundance decreases rapidly, especially for $T>74 \mathrm{MK}$. The opposite is true for ${ }^{27} \mathrm{Al}$ and for ${ }^{26} \mathrm{Mg}$ (which does not appear in the figure): their abundances increase rapidly with $\Delta X$ (at least for $\Delta X<0.1)$ and with $T$.

These trends, namely the stability of $\mathrm{Na}$ with $\Delta X$, the decrease of $\mathrm{Mg}$ (mainly the form of ${ }^{24} \mathrm{Mg}$ ) and the increase of $\mathrm{Al}$ and ${ }^{26} \mathrm{Mg}$, allow one to constrain the range of both $T$ and $\Delta X$. An inspection of Fig. 3 shows that towards the middle of the explored temperature range, between 74 and $76 \mathrm{MK}$, and for consumed $\mathrm{H}$ fraction $\Delta X / X \sim 2-3 \%$, the results of the calculations for the mixture are quite close to the observed extreme values, for all the involved elements. Thus, at least in the framework of our idealised study (constant $T$ ), there is a formal solution to the problem we tackled. Taking into account the various uncertainties of that problem (nuclear reaction rates at such low temperatures, observational errors) and the fact that temperatures do not remain constant in realistic nucleosynthesis sites, we consider this formal solution as extremely satisfactory. We note that the constraints we derived on temperature are rather strong, in view of the strong dependence of nuclear reaction rates on that parameter; however, the physical significance of our derived temperature in the case of convective zones will be analysed in Sect. 5.1. On the contrary, the constraints on $\Delta X$ are obviously unrealistic, since in a realistic astrophysical site, some mixing of material from the H-burning zone with fresh $\mathrm{H}$ (and other metals) from external zones is always expected; $\Delta X$ can be hardly defined then.

\subsection{Mixing of processed with original material}

Once the extreme observed abundances $X_{\mathrm{E}, \mathrm{OBS}}$ are understood (as a result of mixture of one part of the material processed at $T \sim 75 \mathrm{MK}$ and $\Delta X \sim 0.025$ with $\sim 0.30$ parts of the original one), all other observed abundances can be recovered by assuming mixing with various dilution factors (larger than 0.3 ). The results of that exercice appear in Fig. 4.

In the upper panel of Fig. 4 we first display again the results of calculations at $T=74 \mathrm{MK}$, but this time the curves are plotted as a function of the $\mathrm{O}$ abundance; it can be seen that the results of the calculations deviate a lot from the observations (points), as $\mathrm{O}$ decreases rapidly towards its equilibrium value.

Selecting the point corresponding to $\Delta X / X \sim 0.025$ (in the left of all panels of Fig. 4, after the analysis of Sect. 3.2) and mixing that composition with original material to various degrees (dilution factors $f=0.3,0.8,5$. and 40 , respectively) we obtain the series of open symbols in the bottom panels of Fig. 4. Values to the left correspond to $f=0.3$, i.e. $1 / 3$ of original material for every part of processed one. An element heavily depleted in the H-processed zone, like oxygen, will have $\sim 1 / 4$ of its original abundance in the mixture; on the other hand, elements largely overproduced in the H-burning zone, like $\mathrm{Na}$ and $\mathrm{Al}$, will have their processed abundances reduced by $\sim 30 \%$ in that same mixture. Those values (with $f=0.3$ ) correspond to $X_{\mathrm{E}, \mathrm{OBS}}$, as discussed in Sect. 3.3; for larger values of $f$, the composition gets closer and closer to the original one. Overall, the obtained mixtures reproduce quite satisfactorily the full range of observations.

A comparison of the upper and lower panels in Fig. 4 clearly shows the important role of dilution in getting the observed abundances from those in the H-burning zone: the latter (caracterized by extremely low equilibrium values of oxygen) are not 

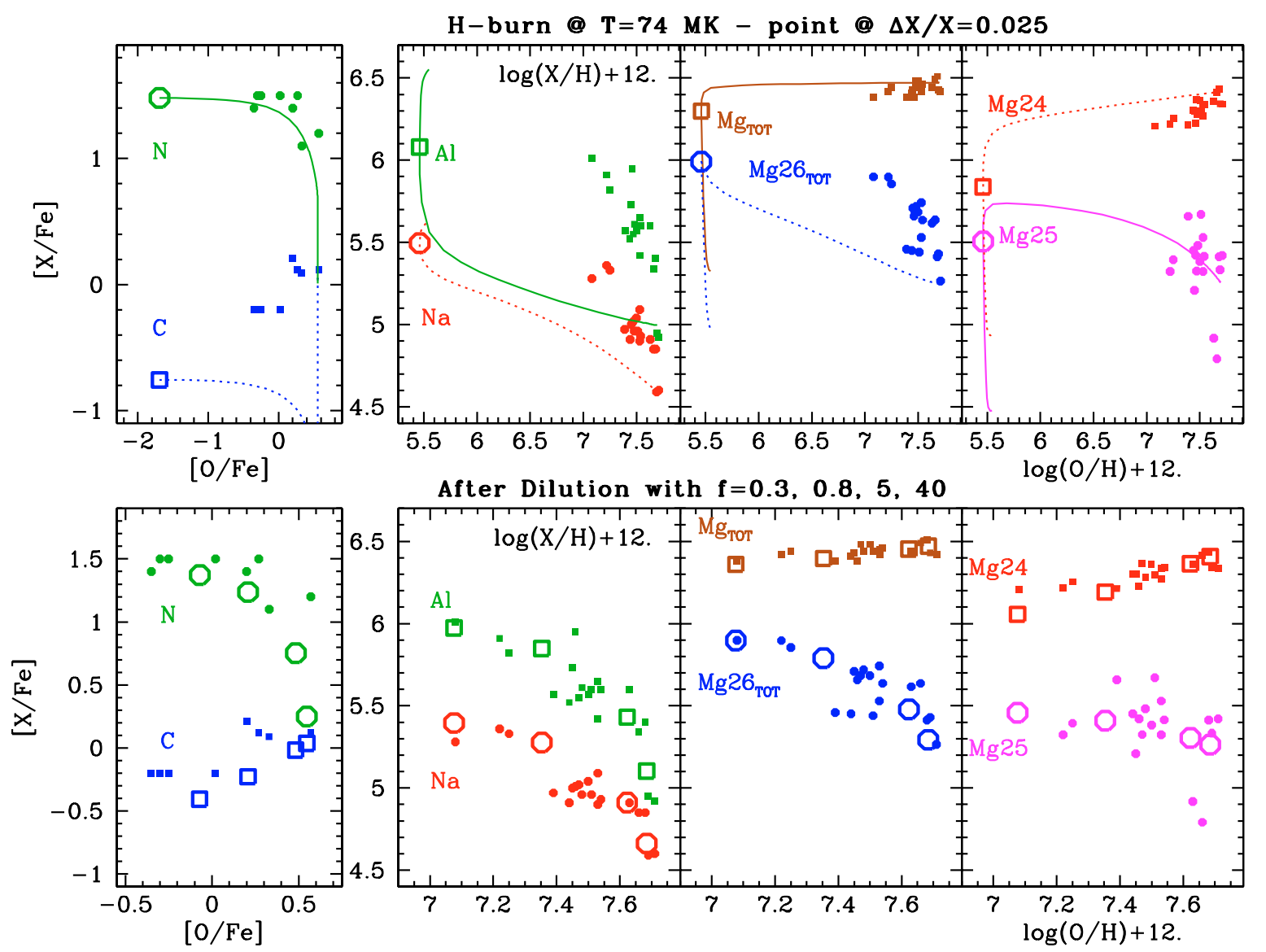

Fig. 4. Abundances of $\mathrm{C}, \mathrm{N}, \mathrm{Na}, \mathrm{Mg}, \mathrm{Al}$ and $\mathrm{Mg}$ isotopes as a function of $\mathrm{O}$ abundance. The $\mathrm{O}$ abundance scale is expanded in the upper row, to accomodate the range of values (i.e. down to the $\mathrm{O}$ equilibrium value) obtained in the calculation at $T=$ const. $=74 \mathrm{MK}$; results of calculations are shown by the solid and dotted curves in each panel, with $\mathrm{O}$ reaching its equilibrium value in the extreme left. Abundances corresponding to a consumed $\mathrm{H}$ fraction of $\Delta X / X_{0}=0.025$ are shown with open circles and squares in each panel in the upper row. Observations (filled points in all panels) are from references given in Fig. 1. Open symbols in the lower row panels denote abundances obtained after mixing the calculated abundances at $\Delta X / X_{0}=0.025$ of the corresponding upper panels with various amounts of material having the original composition. The corresponding dilution factors appear on top of the lower row panels; smaller dilution factors result in abundances closer to the processed ones (e.g. higher $\mathrm{Na}, \mathrm{Al}$ etc.). The abundances of $\mathrm{Mg}_{\mathrm{TOT}}$ and ${ }^{26} \mathrm{Mg}_{\mathrm{TOT}}$ include those of ${ }^{26} \mathrm{Al}$.

at all reminiscent of the observed abundance values, even the most extreme ones. Only when mixing of processed and original material is assumed, the full range of observed values is recovered. In the case of NGC 6752 considered here, the abundances of each and every one of the observed CNONaMgAl elements and $\mathrm{Mg}$ isotopes are reproduced extremely well, for a very restricted range of $\mathrm{H}$-burning temperatures and fraction of $\mathrm{H}$ consumed. It is not clear whether such a highly idealised situation can occur in a realistic site; we discuss that point in Sect. 5.

In Fig. 5 we take a closer look to the situation concerning the $\mathrm{Mg}$ isotopes, since they play a critical role in the determination of the temperature range (due to the decrease of ${ }^{24} \mathrm{Mg}$ at high $T$ and $\Delta X$ ). Indeed, $\mathrm{Al}$ and $\mathrm{Na}$ behave rather similarly (they both increase with $T$ and $\Delta X$ ) and are not sufficiently sensitive to pinpoint $T$.

In Fig. 5 it is seen that, in the selected conditions of $T$ and $\Delta X,{ }^{24} \mathrm{Mg}$ is slightly underproduced w.r.t. observations (by $0.15 \mathrm{dex}$ ), while the ${ }^{26} \mathrm{Mg} /{ }^{24} \mathrm{Mg}$ ratio is slightly overproduced. We note that ${ }^{26} \mathrm{Al}$ is counted in the abundance of ${ }^{26} \mathrm{Mg}$ (and of total $\mathrm{Mg}$, as in Fig. 4) and is at the origin of the large ${ }^{26} \mathrm{Mg} /{ }^{24} \mathrm{Mg}$ ratio; if ${ }^{26} \mathrm{Al}$ is neglected, then the extreme abundances of ${ }^{26} \mathrm{Mg} /{ }^{24} \mathrm{Mg}$ in NGC 6752 are underpredicted by a factor of 2.5 (open squares in Fig. 5).

\section{Lithium and fluorine in globular clusters}

In the previous section, mixing of processed with original material was invoked, in order to explain why the observed oxygen abundances (even the most extreme ones) are far above the processed equilibrium value. In actual reality, this constraint does not require completely unprocessed (i.e. truly pristine) material, but only material processed below $\sim 20 \mathrm{MK}$, i.e. with unaltered abundances of $\mathrm{O}$ and heavier elements. Since a large fraction of the mass of a star is found at temperatures lower than $20 \mathrm{MK}$, our analysis so far does not help clarify the nature of the unprocessed material: is it pure interstellar medium, or can it be just part of the stellar envelope?

A crucial constraint, recently discovered, can help distinguish between the two alternatives. Pasquini et al. (2005) reported observations of $\mathrm{Li}$ in 9 turn-off stars of NGC 6752 with UVES (see Fig. 6) and found that $\mathrm{Li}$ is correlated with $\mathrm{O}$ and anticorrelated with $\mathrm{Na}$. Stars with "original" $\mathrm{O}$ (high) and $\mathrm{Na}$ (low) have a Li content similar to field halo stars in the Spite plateau, i.e. $A(\mathrm{Li})=\log (\mathrm{Li} / \mathrm{H})+12 \sim 2.3$. Stars with "extreme" abundances (lowest $\mathrm{O}$ and highest $\mathrm{Na}$ ) are not devoid of Li but have a significant amount of $A(\mathrm{Li}) \sim 2$. In view of the fragility of $\mathrm{Li}$, which "burns" at temperatures higher than $\sim 2.2 \mathrm{MK}$, this discovery implies that the "unprocessed" material of our mixture 


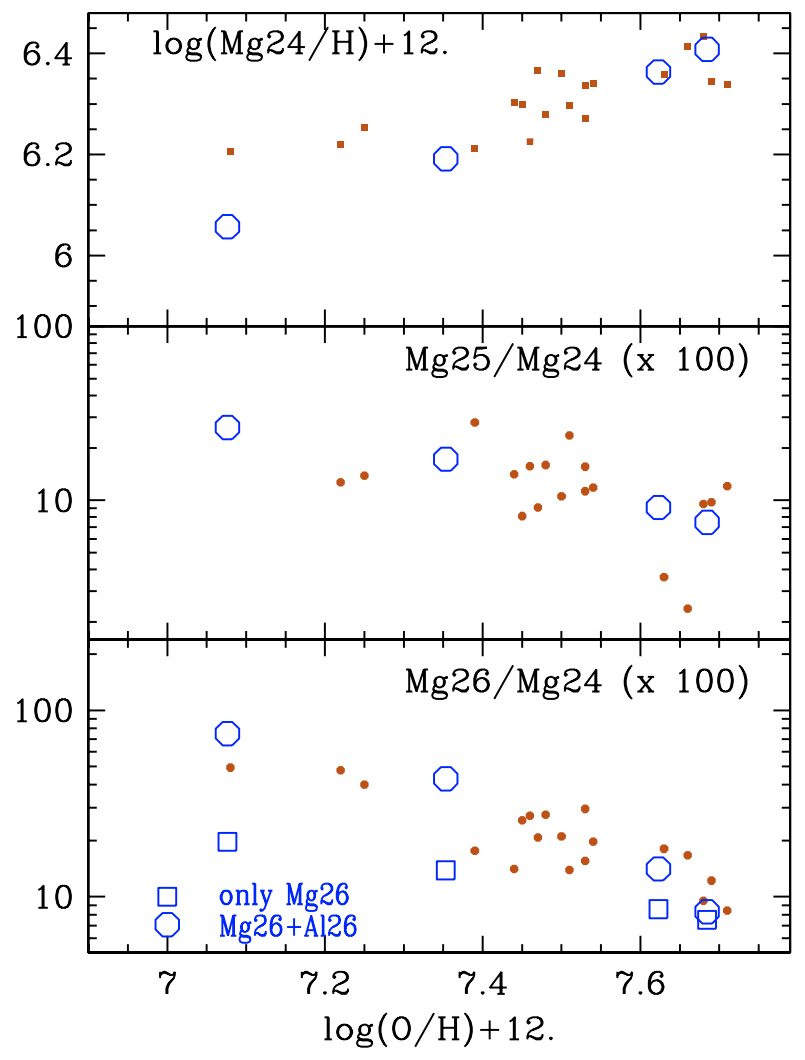

Fig. 5. $\mathrm{Mg}$ isotopic ratios vs. $\mathrm{O} / \mathrm{H}$. Theoretical results (open symbols) are obtained for $T=74 \mathrm{MK}$ and at $\Delta X / X=0.025$, as in Fig. 4; they are mixed to various degrees with material of original composition (mixing factors $f=0.3,0.8,5$ and 40 , from left to right). Observations (filled points) are as in Fig. 1.

has always been kept cooler than 2.2 MK. Since the corresponding stellar zones are a tiny fraction of the mass of any star, it appears natural to conclude that the "unprocessed" material of our mixture is plain ISM.

In Fig. 6 we repeat the procedure followed in Sect. 3.3 and Fig. 4, this time for $\mathrm{Li}$ and $\mathrm{Na}$. Material processed at $T=74 \mathrm{MK}$ (until a fraction of $\mathrm{H} \Delta X / X=0.025$ is consumed) is mixed with $f$ parts of original material, which has $A(\mathrm{Li})=2.65$ (i.e., the cosmological value after WMAP + SBBN; Steigman 2006). By varying $f$ between 0.1 and some high value (above 50) we naturally obtain a decline of the $\mathrm{Li}$ abundance with increased $\mathrm{Na}$ abundance (open circles in Fig. 6). This corresponds to the $\mathrm{Li}$ abundance with which each individual GC star is born. Assuming then that in each star Li has been uniformly depleted by $0.4 \mathrm{dex}$ from its initial value determined previously, as is the case for halo stars in the Spite plateau (e.g., Charbonnel \& Primas 2005), we obtain the filled circles in Fig. 6. This corresponds to the predicted present $\mathrm{Li}$ abundance in the turnoff GC stars that can be directly compared with the observational data.

Pasquini et al. (2005) already interpreted the presence of $\mathrm{Li}$ in the most Na-rich stars of NGC 6752 as an additional clue in favor of the self-enrichment scenario. These authors suggest that $\mathrm{Li}$ must have been created by the same stars that build up the other abundance anomalies, and discuss the possible contribution of AGB stars (see Ventura \& D'Antona 2002a). Our analysis convincingly shows, however, that $\mathrm{Li}$ production by the stellar polluters is not mandatory in the case of NGC 6752 where the abundance patterns are nicely reproduced by mixing to various degrees pristine ISM with H-processed (and totally Li-free)

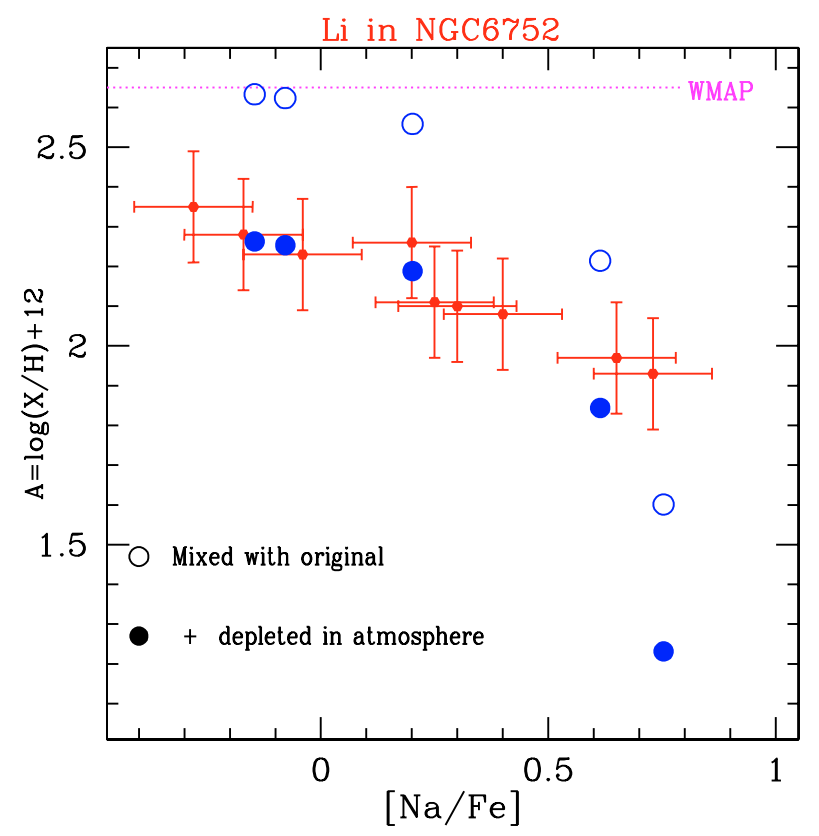

Fig. 6. $\mathrm{Li}$ in the globular cluster NGC 6752. Observations (filled symbols with error bars) are from Pasquini et al. (2005) derived for the Alonso et al. (1996) temperature scale. Open symbols indicate H-burned material ( $\Delta X \sim 0.025$ of $\mathrm{H}$ consumed and no Li present), mixed to various degrees with material of original composition (containing Big Bang Li, at the level of WMAP); dilution factors are 0.1, $0.6,5,40$ and 250, from left to right. Filled symbols indicate uniform depletion of 0.4 dex in all theoretical mixtures, to account for the difference between the WMAP constraint on primordial $\mathrm{Li}$ and observations of the "Spite plateau" (Charbonnel \& Primas 2005).

material. This supports the results of Decressin et al. (2007) who successfully explain the chemical inhomogeneities in NGC 6752 through mixing of the wind of fast rotating massive main sequence stars with pristine material by using a dilution factor derived from the $\mathrm{Li}$ behaviour. Last but not least, the predictions shown in Fig. 6 provide additional support to the idea that old metal-poor low-mass stars manage to destroy part of their initial $\mathrm{Li}$ abundance in a very uniform way, both in the halo and in GCs (Talon \& Charbonnel 2004; Richard et al. 2005; Korn et al. 2006; Charbonnel 2006).

Pasquini et al. (2005) underline the different Li abundance patterns found in NGC 6752 and NGC 6397 and which may be important to pinpoint the processes involved in the selfenrichment scenario. In NGC 6397 indeed the turnoff stars observed to date all share the same $\mathrm{Li}$ abundance with essentially no intrinsic scatter (Pasquini \& Molaro 1996; Thévenin et al. 2001; Bonifacio et al. 2002; but see also Korn et al. 2006), although large variations in $\mathrm{C}, \mathrm{N}, \mathrm{O}$, and Na were uncovered in this GC (Carretta et al. 2005). We note, however, that in NGC 6397 the stars for which both $\mathrm{Li}$ and $\mathrm{Na}$ could be determined (i.e., $[\mathrm{Na} / \mathrm{Fe}]$ between +0.09 and +0.35$)$, cover only a modest range of the Li-Na anticorrelation seen in NGC 6752: they have $[\mathrm{Na} / \mathrm{Fe}]$ between +0.09 and +0.35 , and in this $\mathrm{Na}$ range $\mathrm{Li}$ is also almost constant in NGC 6752 as can be seen in Fig. 6. Li determinations for stars exhibiting stronger $\mathrm{Na}$ enrichment are urgently needed in NGC 6397 as well as in other GCs before one can conclude on possible variations in the self-enrichment scenario from cluster to cluster.

Fluorine, another fragile element (albeit less fragile than Li) has been observed by Smith et al. (2005) in seven red giants of M4, in high resolution infrared spectra obtained with the Gemini 


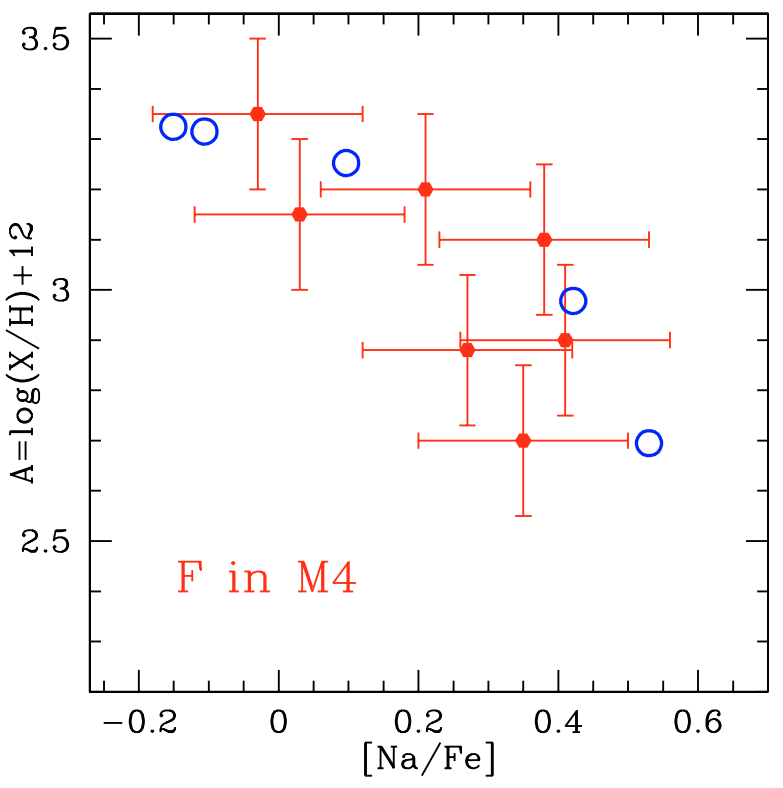

Fig. 7. Fluorine in the globular cluster M4. Observations are from Smith et al. (2005). Open symbols indicate H-burned material at $T=74 \mathrm{MK}$ ( $\Delta X \sim 0.025$ of $\mathrm{H}$ consumed and no $\mathrm{F}$ present), mixed to various degrees with material of original composition; dilution factors are $0.1,0.6$, 5,40 and 250 , from left to right.

South telescope. Fluorine is usually destroyed in high temperature H-burning and its abundance in M4 is again anticorrelated with the one of $\mathrm{Na}$ (Fig. 7): an increase by a factor of $\sim 3$ in $\mathrm{Na}$ corresponds to a decrease of the $\mathrm{F}$ abundance by factor of $\sim 4$. Applying exactly the same prescription as in the case of $\mathrm{Li}$, i.e. by diluting material processed at $74 \mathrm{MK}$ (rich in $\mathrm{Na}$ and having no $\mathrm{F}$ ) with $f$ parts of pristine material (poor in $\mathrm{Na}$ and assumed to have $\log (\mathrm{F} / \mathrm{H})+12=3.35$ ), we obtain the open symbols in Fig. 7. Again, the full range of observed values is (unsurprisingly) reproduced. Smith et al. (2005) interpret the F-Na anticorrelation as the result of pollution of the intracluster gas by relatively massive AGB stars that destroy $F$ in the course of their evolution. We note, however, that the observed fluorine abundance variations do not provide a tight constraint to the mass of the polluters and does not help to discriminate between massive AGB stars and massive stars (see Sect. 5) which both destroy this element in their interior.

The conclusion of this section is that observations of fragile elements ( $\mathrm{Li}, \mathrm{F})$ in GC stars are naturally understood in the framework of the "mixture scenario" explored here. This has already been mentioned in previous works (Pasquini et al. 2005; Decressin et al. 2007) but our study is the first to show it quantitatively. The advantage of those observations (especially Li) is that they clearly indicate that the unprocessed material has to be of pristine nature (i.e. pure ISM) rather than from the stellar envelope of the polluter itself; this information cannot be obtained from the abundance patterns of the heavier elements, analysed in Sect. 3. However, contrary to the case of the heavy elements, observations of fragile elements offer no clue as to the temperature range of the processed material and cannot be used to pinpoint the mass of the stellar polluters.

\section{Temperature and nucleosynthesis sites}

\subsection{H-burning temperature in stars}

The analysis of Sect. 3 is specifically adapted to the case of NGC 6752. In the framework of our extremely simplified model (nucleosynthesis at constant temperature, mixing with pristine material), that analysis allows us to determine a quite narrow range in the $(T, \Delta X / X)$ plane where $\mathrm{H}$-burning products reproduce the full set of observational data for NGC 6752, after appropriate mixing.

The "constraint" on $\Delta X$ is rather meaningless, since in most realistic situations (convective cores of massive stars, bottoms of AGB convective envelopes, see below) hydrogen is constantly mixed in the H-burning zones, both replenishing (at least partially) the $\mathrm{H}$-content of the zone and diluting the abundances of the H-burning products. It is important, however, to find that the observational data are reproduced for the same value of $\Delta X$ (no matter what that value is), just for consistency reasons: if e.g. at a given $T$, the observed Na excess was reproduced only early on (at low $\Delta X$ ) and the observed $\mathrm{Al}$ excess only at late times (large $\Delta X)$, no meaningful conclusions about $T$ would be drawn.

The constraint on temperature $T$ is quite robust, since nuclear reaction rates display such a strong temperature dependence: for instance, for $T<70 \mathrm{MK},{ }^{24} \mathrm{Mg}$ is hardly affected at all, while for $T>80 \mathrm{MK}$ it is rapidly destroyed. Although H-burning temperatures do not remain absolutely constant during stellar evolution, they vary relatively little in quiescent burning stages. It is meaningful then to ask in which kind of realistic stellar environment(s) correspond the temperatures of 74-76 MK that we determined through the analysis of Sect. 3.

Maximum $\mathrm{H}$-burning temperatures corresponding to various stages of stellar evolution are displayed in Fig. 8 as a function of initial stellar mass. The theoretical values are given for models with $[\mathrm{Fe} / \mathrm{H}]=-1.5$. Massive stars (i.e., with an initial mass $\geq 20 M_{\odot}$ ) are from Decressin et al. (2007) while low- and intermediate-mass stars come from Decressin et al. (in preparation) and Siess \& Pumo (2007); for massive AGBs we also show some predictions by Ventura \& D'Antona (2002b). In the case of core H-burning we show the range covered by the central temperature during the whole main sequence (vertical straight lines). For the other phases we show only the maximum temperature reached in the H-burning regions of interest: in the H-burning shell during the red giant phase (i.e., at the "tip" of the RGB; open squares) and during the central He-burning phase (i.e, on the "clump"; open circles), and at the bottom of the convective envelope in AGB or super-AGB stars (filled squares for the models by Decressin et al. and Siess, filled circles for the models by Ventura \& D'Antona).

The horizontal dotted lines enclose the temperature range reproducing the extreme abundances that are observed in NGC 6752, as discussed in Sect. 3. However, this temperature range is not necessarily directly comparable to those of the stellar models. Obviously, for nuclear burning in radiative (and narrow) regions, the temperature of our calculations is simply the temperature of those regions. But for convective regions (such as the cores of massive stars or the Hot-Bottom burning envelopes of AGBs) the situation is different: burning takes place essentially in the hottest region (the massive star center or the bottom of the AGB envelope) but it involves fuel from all over the convective region (because convective timescales are shorter than nuclear ones). In those conditions it can be shown that the fuel burns with an effective reaction rate

$R_{\text {eff }}=\frac{1}{M} \int R(T(m)) \mathrm{d} m$

where $R(T(m))$ is the reaction rate at the shell of mass coordinate $m$ and temperature $T(m), \mathrm{d} m$ is the mass of that shell and $M$ the total mass of the convective region (see e.g. Eq. (5) in Prantzos et al. 1986). In that integral, the largest contribution 


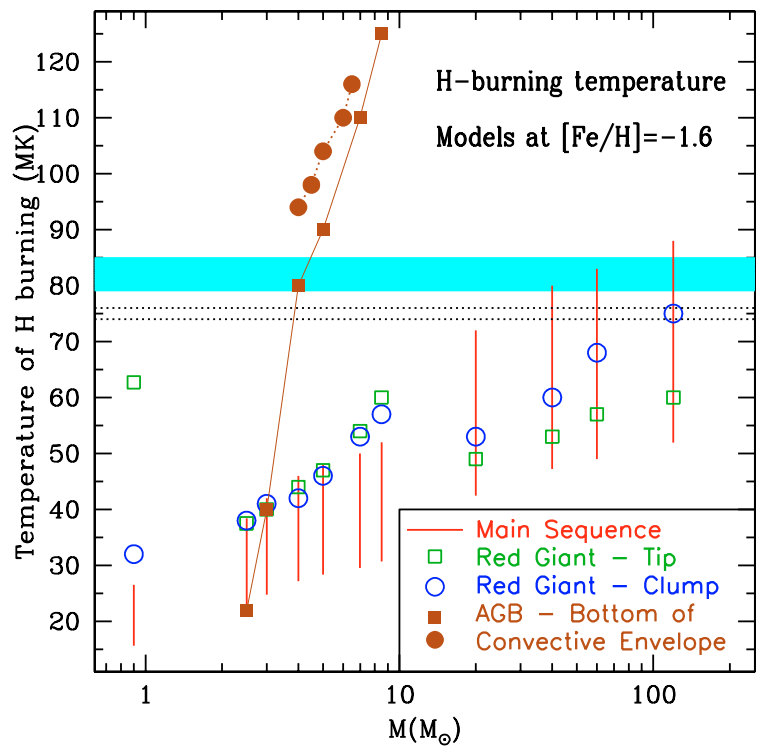

Fig. 8. H-burning temperatures at various phases of a star's life, as a function of initial stellar mass. The correspondence between symbols and evolutionary phases is displayed in the bottom right part of the figure. Vertical straight lines (for central H-burning) start at the ZAMS and end at central H-exhaustion. For the other phases we show only the maximum temperature reached in the H-burning zones of interest (see details in the text). The two horizontal dotted lines enclose the temperature range of our one-zone models reproducing the extreme abundances that are observed in NGC 6752, after the analysis of Sect. 3. The shaded aerea encloses our rough estimates for the corresponding maximal temperature range in the case of convective $\mathrm{H}$-burning regions (in the bottom of AGB envelopes or in the center of massive star cores), as discussed in Sect. 5.1.

comes from the hottest (highest $T$ ) region (because reaction rates are extremely sensitive to temperature and contributions from all cooler regions may be considered negligible), but multiplication by $\mathrm{d} m / M$ leads then to a lower effective rate, corresponding to lower $T$.

Our calculated rates obviously simulate $R_{\text {eff }}$ of convective regions, and thus allow us to constrain only the temperature $T_{\mathrm{eff}}$ which corresponds to $R_{\text {eff }}$. This is only a lower limit to the actual temperature $T_{\mathrm{b}}$ where burning takes place (i.e. the star center or the bottom of the AGB envelope). How much higher may that temperature $T_{\mathrm{b}}$ be? It depends on the actual temperature profile of the convective region. An approximate estimate can be made as follows:

Assuming that $R(T) \propto T^{L}$ (with e.g. $L=20$ for $\mathrm{CNO}$ cycle reactions) and that $\mathrm{d} m\left(T_{\mathrm{b}}\right)=M / N$ (the hottest shell of temperature $T_{\mathrm{b}}$ has a mass $\mathrm{d} m$ which is $N$ times smaller than the mass $M$ of the convective region) one finds that: $T_{\mathrm{b}} / T_{\mathrm{eff}}=N^{1 / L}$. For instance, for $L=20$ and $N=10$, one has $T_{\mathrm{b}} / T_{\text {eff }}=10^{0.05} \sim 1.12$. Thus, our $T_{\text {eff }}=74 \mathrm{MK}$ corresponds to a more "realistic" peak temperature of the convective region $T_{\mathrm{b}}=83 \mathrm{MK}$. Since $\mathrm{NeNaMgAl}$ nuclei have higher Coulomb barriers than CNO nuclei, $L$ must be slightly larger than 20 and $T_{\mathrm{b}}$ slightly lower than derived above.

Our conclusion then is that the actual maximum temperature of convective H-burning zones which leads to the observed extreme abundances in NGC 6752 is $\sim 10-15 \%$ higher than the temperature determined here, i.e. in the $80-85 \mathrm{MK}$ range. Note, however, that reaction rate uncertainties also impact on that derivation.

\subsection{Low-mass stars presently observed in GCs}

As already mentioned, the temperature inside the GC turnoff stars that we observe today (with a typical mass of the order of $0.8-0.9 M_{\odot}$, see the corresponding vertical line in Fig. 8) is too low for the proton-capture reactions to operate in the $\mathrm{NeNa}$ and $\mathrm{Mg}-\mathrm{Al}$ chains, implying that the observed abundance variations cannot be of intrinsic nature. While low-mass stars climb the RGB, the temperature increases inside the H-burning shell surrounding the degenerate $\mathrm{He}$-core; it reaches relatively high values (of the order of $\sim 60 \mathrm{MK}$ for the $0.9 M_{\odot}$ model) that are sufficient for the NeNa-chain to operate and for the two heavier $\mathrm{Mg}$ isotopes to burn around the H-burning shell deep inside the star (Kudryashov \& Tutukov 1988; Denisenkov \& Denisenkova 1989, 1990; Langer et al. 1993; Langer \& Hoffman 1995; Cavallo et al. 1999; Weiss et al. 2000). However, the observed variations of ${ }^{24} \mathrm{Mg}$ require still higher internal temperature (see also Arnould et al. 1999). These difficulties, together with the finding that the GC chemical patterns (i.e., anticorrelations) are similar in turnoff and RGB stars, imply that the abundance anomalies cannot be generated in the course of the evolution of the longlived objects that we are currently observing. In other words, the so-called evolution hypothesis that calls for non-canonical very deep mixing inside the low-mass star itself should be discarded.

\section{3. $A G B$ stars}

It is usually claimed that hot bottom burning (hereafter HBB) in massive AGB stars undergoing thermal pulses is responsible for the observed composition anomalies in GCs. As can be seen in Fig. 8 indeed, the relatively high $\mathrm{H}$-burning temperatures required to reproduce the extreme NGC 6752 abundances are encountered at the bottom of the convective envelope of these objects. The region in the envelope that is hot enough for $\mathrm{H}$-burning is relatively thin; however, thanks to the very efficient convective mixing, the matter in the entire envelope goes through the burning region a large number of times $(\sim 1000)$ during each thermal pulse cycle. This directly affects the surface and wind composition of the stars. Note that for a given initial stellar mass the temperature at the bottom of the convective envelope $T_{\text {bce }}$ increases from pulse to pulse on the AGB and reaches the maximum value shown in Fig. 8 after a few thermal pulses (see e.g., Ventura $\&$ D'Antona 2002a). As the star evolves and loses mass $T_{\text {bce }}$ decreases again.

The difference in the theoretical predictions of the two sets of models considered here underlines the uncertainties associated with the input physics that is adopted in the computations. Here it comes mainly from one of the most relevant uncertainties connected to stellar evolution, i.e., the treatment of convection that strongly influences the stellar characteristics during the TPAGB phase: Decressin and Siess models use the traditional MLT (Mixing Length Theory) while Ventura \& D'Antona use the FST (Full Spectrum of Turbulence; Canuto et al. 1996). In the latter case the convection efficiency is stronger, leading to higher temperatures at the bottom of the convective envelope of the AGBs (see Ventura \& D'Antona 2002a for a more detailed discussion). Other key (but uncertain) input parameters of the AGB models such as the mass loss prescription also influence the theoretical predictions and are important for nucleosynthesis purposes (see e.g., Forestini \& Charbonnel 1997).

The present simple analysis precludes us from deriving further conclusions that require sophisticated and complete stellar evolution computations. During the AGB phase the surface and wind composition of the stars depend indeed on the 
subbtle competition between $\mathrm{HBB}$ and third dredge-up. As a matter of fact, detailed studied based on custom-made AGB models pointed out several severe drawbacks of the AGB pollution scenario (Ventura el al. 2001, 2002; Denissenkov \& Herwig 2003; Karakas \& Lattanzio 2003; Herwig 2004a,b; Ventura \& D'Antona 2002a,b,c; Karakas et al. 2006). These difficulties come from the fact that the third dredge-up does contaminate the AGB envelope with the products of helium burning $\left({ }^{16} \mathrm{O}\right.$ and ${ }^{25,26} \mathrm{Mg}$ in particular) and creates abundance patterns in conflict with the ones observed (see e.g., Fenner et al. 2004; Charbonnel 2005, and PC06). In particular, the sum $\mathrm{C}+\mathrm{N}+\mathrm{O}$ does not remain constant in AGB processed material ${ }^{6}$, in contrast to observational requirements: whenever the relevant data are available simultaneously, the sum $\mathrm{C}+\mathrm{N}+\mathrm{O}$ (as well as $\mathrm{Mg}+\mathrm{Al}$ ) is indeed found to be constant from star-to-star within the observational errors (Dickens et al. 1991; Ivans et al. 1999). PC06 discuss other shortcomings of the AGB scenario, related e.g. to the peculiar initial mass function it requires; in addition, they underline the fact that the AGB scenario gives no satisfactory answers as to the role of stars more massive and less massive than the presumed polluters and does not provide a mechanism to trigger the formation of the chemically peculiar low-mass stars that we observe today in GCs.

\subsection{Massive stars}

Finally we see from Fig. 8 that the central temperature of massive main sequence stars (i.e., with an initial mass slightly higher than $40 M_{\odot}$ ) reaches the values required to produce the observed abundance anomalies. In Decressin et al. (2007; see also PC06) we suggest that fast rotation near the breakup allows massive stars to eject their H-burning products through slow winds. The theoretical surface and wind composition of fast rotating massive stars presents thus the H-burning signatures observed in GC stars. A difficulty within this framework concerns the destruction of ${ }^{24} \mathrm{Mg}$ and the related amplitude of the $\mathrm{Mg}$ - $\mathrm{Al}$ anticorrelation; they require indeed a large increase of the ${ }^{24} \mathrm{Mg}(\mathrm{p}, \gamma)$ reaction rate around $50 \mathrm{MK}$ with respect to the published values in order to be fully reproduced in complete stellar evolution models. This is due to the fact that the temperature necessary to efficiently destroy ${ }^{24} \mathrm{Mg}$ is reached in the core of massive stars only at the very end of their main sequence evolution (see Fig. 7 of Decressin et al. 2007). As mentioned in Sect. 3.2, the ${ }^{24} \mathrm{Mg}(\mathrm{p}, \gamma)^{25} \mathrm{Al}$ reaction has a small rate error in the temperature range we consider (see Powell et al. 1999). Note, however that a low-lying resonance cannot be totally excluded (although, based on the known level structure in these nuclei, no resonance is predicted).

For the moment we stress that the Winds of Fast Rotating Massive Stars scenario presents a very interesting framework for the understanding of the self-enrichment of GCs. Fast rotation ${ }^{7}$ loads the surface layers as well as the stellar wind with the appropriate H-burning products. It helps removing that material from the stellar surface through low-velocity stellar winds which can be easily kept within the GC. We note in addition that the abundance patterns are well reproduced when assuming mixing of the

\footnotetext{
${ }^{6}$ Note however that Ventura \& D'Antona (2002a) have managed to keep the total $\mathrm{C}+\mathrm{N}+\mathrm{O}$ abundance within a factor of $\sim 3$ in their AGB models computed with the FST model for convection and with some "extra-mixing" at the base of the stellar convective envelope.

${ }^{7}$ By fast rotation we mean an initial rotation velocity such that the star reaches the so-called critical velocity early on the main sequence. As an example, this corresponds to $400 \mathrm{~km} \mathrm{~s}^{-1}$ on the zero age main sequence for a $60 M_{\odot}$ star.
}

massive star ejecta with pristine gas as required by the extreme observed value of Li in Na-rich stars. Last but not least, massive stars can, through the SN shocks or the ionisation front they produce, trigger star formation in their vicinity (PC06). However, in that case also (as in the case of the AGBs), the IMF of the first generation stars has to be flatter than a normal IMF in order to explain observations (PC06, Smith 2006).

\section{Summary}

In this work, we study quantitatively the nucleosynthetic processes that may be at the origin of the peculiar abundance patterns observed in stars of GCs. Our analysis is adapted, in particular, to the case of NGC 6752, for which a large body of observational data is available. The data, presented in Sect. 2, concern abundances of $\mathrm{C}, \mathrm{N}, \mathrm{O}, \mathrm{Na}, \mathrm{Mg}$ and $\mathrm{Al}$, as well as $\mathrm{Mg}$ isotopes, observed in about two dozen stars of that GC.

In order to constrain the physical conditions (i.e. the temperature range of the H-burning) that gave rise to the observed abundance patterns, we perform nucleosynthesis calculations at constant $T$. We adopt "realistic" initial abundances (i.e. corresponding to field halo stars of similar $[\mathrm{Fe} / \mathrm{H}]=-1.5$ as NGC 6752), a detailed nuclear reaction network and state-ofthe-art nuclear reaction rates (presented in Sect. 3.1).

We find that there exists a rather narrow temperature range around $T \sim 75 \mathrm{MK}$ where the observed extreme abundances of all elements and isotopes in NGC 6752 is nicely co-produced. This happens only after mixing of the nuclearly processed material with $f \sim 30 \%$ of material of pristine composition (Sect. 3.3). This mixing factor is imposed by the fact that the observed extreme oxygen abundance in NGC 6752 is only $\sim 5-7$ times lower than the original one, and never as low as in H-burning regions. In fact, none of the abundances in H-burning zones corresponds to the extreme observed ones; the latter are recovered only after mixing with $30 \%$ of pristine material. We note that in the framework of our study (which assumes no enhancement of the starting abundances for any of the involved nuclei, just field halo star composition) the observed ${ }^{26} \mathrm{Mg}$ excess in NGC 6752 can only be understood in terms of its production as ${ }^{26} \mathrm{Al}$.

We show then quantitatively how, by increasing the mixing factor $f$, one can naturally obtain the full range of observed abundances in NGC 6752, i.e. the well-known anti-correlations between $\mathrm{N}$ and $\mathrm{O}, \mathrm{O}$ and $\mathrm{Na}$ or $\mathrm{O}$ and $\mathrm{Al}$; the behaviour of the $\mathrm{Mg}$ isotopes is also satisfactorily reproduced (Sect. 3.4).

Mixing of processed with pristine material can also naturally explain observed anticorrelations of the fragile elements $\mathrm{Li}$ and $\mathrm{F}$ vs. Na in GCs; this is demonstrated quantitatively in Sect. 4, based on our results for $\mathrm{Na}$ production. Observations of heavy elements constrain the temperature range for H-burning and suggest mixing, but leave unclear the origin of the mixed material (ISM or from the stellar envelopes); observations of the fragile light elements clearly point to ISM origin for the mixed material, since the fraction of the stellar envelopes with $T<2.2 \mathrm{MK}$ (necessary to preserve Li) is negligible. We also note that our analysis supports the conclusion that NGC 6752 turnoff stars did undergo a uniform depletion of their original surface Li abundance similar to that of field halo counterparts. It shows finally that $\mathrm{Li}$ production by the stars that build up the other abundance anomalies is not mandatory in the case of NGC 6752.

Finally, in Sect. 5 we discuss the temperature constraint found in our analysis, which we consider to be quite robust, in view of the sensitivity of nuclear reaction rates to temperature. We note that, in the case of convective regions (such as 
AGB envelopes and massive star cores) the temperature we determined through our analysis is only an effective temperature (corresponding to an average reaction rate over the whole convective region) and provides a lower limit to the actual maximum temperature of the convective zone. We argue that, in view of the temperature dependence of the reaction rates, the maximal temperature of the convective zone should be 10-15\% higher than the one-zone temperature of our models, i.e. in the $80-85 \mathrm{MK}$ range. We find that such temperatures are encountered in the two main candidate polluters, namely massive AGB stars (with HBB) and the most massive stars (above $40 M_{\odot}$ ) on the main sequence. We note, however, that the precise $T$ values are much more model dependent in the former case than in the latter.

Acknowledgements. We thank the anonymous referee for constructive comments. We are indebted to T. Decressin, L. Siess and P. Ventura for providing us with some information on their stellar models.

\section{References}

Alonso, A., Arribas, S., \& Martínez-Roger, C. 1996, A\&A, 313, 873

Angulo, C., Arnould, M., Rayet, M., et al. 1999, Nucl. Phys. A, 656, 3

Arnould, M., Goriely, S., \& Jorissen, A. 1999, A\&A, 347, 572

Bonifacio, P., Pasquini, L., Spite, F., et al. 2002, A\&A, 390, 91

Canuto, V. M., Goldman, I., \& Mazzitelli, I. 1996, ApJ, 473, 550

Carretta E., Gratton, R. G., Lucatello, S., Bragaglia A., \& Bonifacio, P. 2005, A\&A, 441, 131

Carretta E., Bragaglia A., Gratton R. G., Lucatello S., \& Momany, Y. 2007, A\&A, 464, 927

Cavallo, R. M., Sweigart, A. V., \& Bell, R. B. 1999, ApJ, 492, 575

Chafa, A., Tatischeff, V., Aguer, P., et al. 2005, Phys. Rev. Lett., 95, 031101

Charbonnel, C. 1994, A\&A, 282, 811

Charbonnel, C. 2005, From lithium to uranium: Element tracers of early cosmic evolution, ed. V. Hill, P. François, \& F. Primas, (Cambridge Univ. Press), IAU Symp., 228, 347

Charbonnel, C. 2006, Nature, 442, 636

Charbonnel, C., \& Primas, F. 2005, A\&A, 442, 961

Charbonnel, C., Brown, J. A., \& Wallerstein, G. 1998, A\&A, 332, 204

Cottrell P. L., \& Da Costa, G. S. 1981, ApJ, 245, L79

D’Antona, F., Caloi, V., Montalban, J., Ventura P., \& Gratton, R. 2002, A\&A, 395,69

Decressin, T., Meynet, G., Charbonnel C., Prantzos, N., \& Ekström, S. 2007, A\&A, 464, 1029

Denisenkov, P. A. \& Denisenkova S. N. 1989, A. Tsir., 1538, 11

Denisenkov, P. A. \& Denisenkova S. N. 1990, SvA Lett., 16, 275

Denissenkov, P. A. \& Herwig, F. 2003, ApJ, 590, 99

Dickens, R. J., Croke, B. F. W., Cannon, R. D., \& Bell, R. A. 1991, Nature, 351, 212

Fenner, Y., Campbell, S., Karakas, A. I., Lattanzio, J. C., \& Gibson, B. K. 2004, MNRAS, 353, 789

Forestini, M., \& Charbonnel, C. 1997, A\&AS, 123, 241

Fox, C., Iliadis, C., Champagne, A. E., et al. 2005, Phys. Rev. C, 71, 055801

Goswami, S., \& Prantzos, N. 2000, A\&A, 359, 191
Gratton, R. G., Sneden, C., Carretta, E., \& Bragaglia, A. 2000, A\&A, 354, 169 Gratton, R., Bonifacio, P., Bragaglia, A., et al. 2001, A\&A, 369, 87 Gratton, R., Sneden, C., \& Carretta, E. 2004, ARA\&A, 42, 385

Hanson, R. B., Sneden, C., Kraft, R. P., \& Fullbright, J. 1998, AJ, 116, 1286

Herwig, F. 2004a, ApJ, 605, 425

Herwig, F. 2004b, ApJS, 155, 651

Iliadis, C., Iliadis, C., Champagne, A. E., et al. 2001, ApJS, 134, 151

Ivans, I. I., Sneden, C., Kraft, R. P., et al. 1999, AJ, 118, 1273

Izzard, R. G., Lugaro, M., Karakas, A. I., Iliadis, C., \& van Raai, M. 2007, A\&A, 466,641

Karakas, A. I., \& Lattanzio, J. C. 2003, PASA, 20, 279

Karakas, A. I., Fenner, Y., Sills, A., Campbell, S. W., \& Lattanzio, J. C. 2006, ApJ, 652, 1240

Korn, A. J., Grundahl, F., Richard, O., et al. 2006, Nature, 442, 657

Kudryashov, A. D., \& Tutukov, A. V. 1988, Astron. Tsirk., 1525, 11

Langer, G. E., \& Hoffman, R. 1995, PASP, 107, 1177

Langer, G. E., Hoffman, R., \& Sneden, C. 1993, PASP, 105, 301

Mishenina, T. V., Bienaymé, O., Gorbaneva, T. I., et al. 2006, A\&A, 456, 1109

Palacios, A., Bolmont, J., Charbonnel, C., \& Thévenin, F. 2002, 281, Ap\&SS, 213

Palacios, A., Charbonnel, C., Talon, S., \& Siess, L. 2006, A\&A, 453, 261

Pasquini, L., \& Molaro, P. 1996, A\&A, 307, 761

Pasquini, L., Bonifacio, P., Molaro, P., et al. 2005, A\&A, 441, 549

Powell, D. C., Iliadis, C., \& Champagne, A. E. 1999, Nucl. Phys. A, 660, 349

Prantzos, N., Doom, C., Arnould, M., \& De Loore, C. 1986, ApJ, 304, 695

Prantzos, N., \& Charbonnel, C. 2006, A\&A, 458, 135, PC06

Pritzl, B. J., Venn, K. A., \& Irwin, M. 2005, AJ, 130, 2140

Richard, O., Michaud, G., \& Richer, J. 2005, ApJ, 619, 538

Rowland, C., D’Auria, J. M., Starrfield, S., et al. 2004, ApJ, 615, L37

Runkle, R. C., Champagne, A. E., \& Engel, J. 2001, ApJ, 556, 970

Runkle, R. C., Iliadis, C., Champagne, A. E., et al. 2005, Phys. Rev. Lett., 94, 082503

Siess, L., \& Pumo, M. L. 2007, in preparation

Smith, V. V. 2004, in Origin and Evolution of the Elements, ed. A. McWilliam and M. Rauch, Carnegie Obs. Astrophys. Ser. 4 (Cambridge: Cambridge Univ. Press), 186

Smith, G. H. 2006, PASP, 118, 1225

Smith, G. H., \& Norris, J. 1982, ApJ, 254, 594

Smith, V. V., Cunha, K., Ivans, I. I., et al. 2005, ApJ, 633, 392

Sneden, C. 2005, on From lithium to uranium: Element tracers of early cosmic evolution, (Cambridge Univ. Press), ed. V. Hill, P. François, \& F. Primas, IAU Symp., 228, 337

Spite, M., Cayrel, R., \& Plez, B. 2005, A\&A, 430, 655

Steigman, G. 2006, Inter. J. Mod. Phys. E, 15, 1

Talon, S., \& Charbonnel, C. 2004, A\&A, 418, 1051

Thévenin, F., Charbonnel, C., de Freitas Pacheco, J. A., et al. 2001, A\&A, 373, 905

Ventura, P., \& D'Antona, F. 2005a, A\&A, 431, 279

Ventura, P., \& D'Antona, F. 2005b, A\&A, 439, 1075

Ventura, P., \& D'Antona, F. 2005c, ApJ, 635, L149

Ventura, P., D’Antona, F., Mazzitelli, I., \& Gratton, R. 2001, ApJ, 550, L65

Ventura, P., D'Antona, F., \& Mazzitelli I. 2002, A\&A, 393, 215

Weiss, A., Denissenkov, P. A., \& Charbonnel, C. 2000, A\&A, 356, 181

Yong, D., Grundahl, F., Lambert, D. L., Nissen, P. E., \& Shetrone, M. D. 2003, A\&A, 402, 985

Yong, D., Aoki, W., \& Lambert, D. 2006, ApJ, 638, 1018 